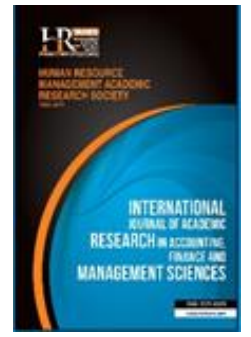

International Journal of Academic Research in Accounting, Finance and Management Sciences

Vol. 10, No.2, April 2020, pp. 133-139

E-ISSN: 2225-8329, P-ISSN: 2308-0337

(c) 2020 HRMARS

www.hrmars.com

To cite this article: Anghel, M.-G., lacob, S. V., Dumbrava, G.-S. (2020). The Calculation of the Price without Arbitration and the Use of This in the Study of the Capital Market, International Journal of Academic Research in Accounting, Finance and Management Sciences 10 (2):133-139

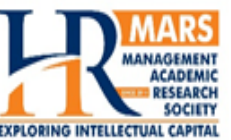

\title{
The Calculation of the Price without Arbitration and the Use of This in the Study of the Capital Market
}

\author{
Madalina-Gabriela Anghel ${ }^{1}$, Ștefan Virgil lacob ${ }^{3}$, Gabriel Ștefan Dumbrava ${ }^{3}$ \\ 1"Artifex" University of Bucharest, Romania, \\ ${ }^{1}$ E-mail: madalinagabriela_anghel@yahoo.com, ${ }^{2} E$-mail: stefaniacob79@yahoo.com \\ ${ }^{3}$ The Bucharest University of Economic Studies, Romania, ${ }^{3} E$-mail: stefan.dumbrava@gmail.com
}

\begin{abstract}
In this article, the authors focused on creating a model for analyzing assets and decisions that can be taken in studying and forecasting the capital market under the impact of inflation. The prices of the capital market can be established on the basis of the normal evolution of deflated prices or based on a study regarding the previous stage and evolution of the capital market. In the capital market, prices can be expressed at market developments or, at other times, at prices without arbitrage. The intertemporal accumulation model of assets is clearly under the influence of capital risk. Therefore, the dynamics of the price evolution rate must play an important role, but this can only be done in close correlation with the dynamics of the inflation rate. Many hypotheses can be considered that happen in the short, medium or long term, in the evolution of prices on the capital market. We say the evolution of prices because, according to this, they coordinate, correlate the investors' intentions to place the capital market assets, to station or to restrict them. From this point of view, any analysis of the forecast, the forecast of the capital market must be done in close accordance with the concrete analysis of the dynamics of inflation. Inflation, in its dynamics, also helps to establish prices on the capital market and, consequently, the returns obtained on the capital market. The market prices are those that determine the returns that can be obtained by investors who place their assets on the capital market. The evolution of prices on the capital market and the evolution of the perspective of capital prices must be closely observed in line with the dynamics of the inflation rate. By assigning assets under risk aversion, it is clear that it is the parameter that highlights the effect and direction in which the investments in the capital market develop. Consequently, we can specify that the dynamics of inflation over a period of time in which the assets are placed is what determines the size of the respective returns. Without a correct estimate of inflation, a certain forecast cannot be made regarding the evolution of the price and, consequently, of the evolution of the returns that happen on the capital market. The dynamics of inflation, determined by statistical-econometric methods, ensure the possibility of the capital market reacting positively or not. Inflation needs to be updated through forecast studies and then used to deflate capital market indicators so that deflated indicators can suggest the forecast of the capital market evolution. In other ideas, we can specify that, the estimation of prices by arbitrary way, as well as the correlative study between the evolution of the activity in the capital markets and the yields that will be obtained are in close agreement. The study also aims at forecasting the capital market in the short, medium and long term, depending on the evolution of prices, the dynamics of inflation during the forecast period.
\end{abstract}

Key words Inflation, Evolution, Capital Market, Inflation Dynamics, Prices Without Arbitrage.

\section{Received: 20 May $2020 \quad$ C The Authors 2020}

Revised: 25 Jun $2020 \quad$ Published by Human Resource Management Academic Research Society (www.hrmars.com)

Accepted: $\quad 01 \mathrm{Jul} 2020 \quad$ This article is published under the Creative Commons Attribution (CC BY 4.0) license. Anyone may Published Online: 24 Jul 2020 reproduce, distribute, translate and create derivative works of this article (for both commercial and non-commercial purposes), subject to full attribution to the original publication and authors. The full terms of this license may be seen at: http://creativecommons.org/licences/by/4.0/legalcode 


\section{Introduction}

In the article on estimating the evolution of prices without arbitrage and the dynamics of inflation, the authors considered determining the factors that influence the stability and evolution of the capital market. The capital market, in a stabilized economy, plays an important role in the harmonized evolution of the national economy. The analysis of the capital market can be carried out taking into account the dynamics of inflation, which is clearly correlated with the evolution of the dynamics of the yields of assets, stocks, based on which a study can be made based on the considered factors, so that, finally, we determine precisely the allocation of assets under normal conditions or risk aversion.

In the study, the authors highlighted the possibility of evaluating the evolution of the prices of the capital market, the inflation rate as a forecast, in the context in which the interest of the investors in the capital market is precisely determined by the way in which there is the prospect of materializing the investments through a high yield. The analysis of the correlation between the dynamics of the inflation rate and the dynamics of yields cannot be done without a thorough study. This analysis cannot be carried out by chance, the basic element being the estimation of the dynamics of inflation, being considered in this way a model, which would lead to the possibility of determining some parameters, which would identify the prospects for movement in the capital market.

It should be borne in mind that a positive evolution of the capital market is made according to the way the inflation shock correlates with the expected prospects of the investors. A positive correlation between the dynamics of the inflation rate and the prospect of returns must be the element that underlies the study of the capital market. Asset allocation is correlated with the investment horizon, but in line with the inflation rate correlation, which should ensure a real possibility to identify the effect of this destabilizing factor of the market in general and not just the capital market.

The model in which the intertemporal portfolio will be built and analyzed, in the perspective of the evolution of assets and possible decisions, cannot be and cannot avoid the effect of the inflation rate. In general terms, the structural analysis over time of nominal yields should be based on a thorough study, based on regression models, which offers the perspective of interpreting how the capital market evolves closely in line with the dynamics of inflation, which may to take place from the short, medium or long term, in which the analysis of the capital market is considered.

The dynamics of inflation is important in the sense that this is a determining factor in determining the value of assets and portfolios on the capital market. The estimation of the dynamics of inflation must be made in close connection with the estimation of the dynamics of the returns of stocks and assets, so that, finally, decisions regarding market placements can be made, depending on the risk that the level recorded in the inflation rate or the evolution of prices may entail. The model regarding the estimation of prices through non-arbitrage methods and the correlation of the dynamics of inflation with the evolution of the returns is a problem that is within the reach of the researchers in the desire to establish the perspective and the efficiency of investments in the capital market.

\section{Literature review}

Amini et al. (2010) were concerned about the evolution of stock market returns. Anghelache \& Anghel (2018) studied the main methods and models used in capital market analyzes. Aruoba (2019) analyzed a number of methods of estimating inflation and the real interest rate. Cooper (2009) addressed the elements of risk premiums. Dobrodolac (2011) presented a series of econometric models applied in the real economy. Dougherty (2008) is a reference work in the field of econometrics. Elliott et al. (2015) highlighted the importance of the null hypothesis in statistical testing. Ferreira \& Santa-Clara (2011), as well as Hjalmarsson (2010) were concerned with the methods of estimating the return on the capital market. Giacomini \& Rossi (2010) conducted a study on forecasts in unstable environments. Jarrow \& Yildirim (2003) analyzed the evolution of the prices of securities under inflation conditions. Markowitz (2014) addressed issues related to average variation. Piketty (2014) studied some issues related to capital, understanding the dynamics of capitalism, as well as the relationship between growth and return on capital. Rapach \& Zhou (2013) investigated aspects regarding to estimating the profitability of the shares. Wachter (2003) analyzed the correlation between risk aversion and long-term debt allocation. Welch \& 
Goyal (2008) conducted a study on estimating equity premiums. Zhou (2010) was concerned with the study of the efficiency of models based on asset prices, conducting an empirical study in this regard.

\section{Methodology of research, data, results and discussions}

\subsection{The expression at the price without arbitrage}

The expression of the assets in the market price can also be done by arbitrage, thus understanding a price determined by the effect of the competition on the market. Also, the price can be expressed at the real price of the bonds.

Applying the Lema concept in the working relationship, we can write the nominal yield of the bond, using the relation:

$$
\frac{d P_{n}\left(r_{t}, \pi_{t}, t, T\right)}{p_{n}\left(r_{t}, \pi_{t}, t, T\right)}=\mu_{n}(t, T-t) d t-B_{n r}(T-t) g_{t} d W_{t}^{r}-B_{n \pi}(T-t) g_{\pi} d W_{t}^{\pi}
$$

Where:

$$
\begin{aligned}
& \tau=T-t \\
& \left.\left.\mu_{n}(t, \tau)=\frac{d}{d \tau} A_{n}(\tau)+\frac{d}{d \tau} B_{n r}(\tau) r_{t}+\frac{d}{d \tau} B_{n \pi}(\tau) \pi_{t}-B_{n r}(\tau) k_{r} \overline{(r}-r_{r}\right)-B_{n \pi}(\tau) k_{r} \overline{(\pi}-\pi_{r}\right)+ \\
& \frac{1}{2}\left(B_{n r}(\tau)^{2} g_{r}^{2}+2 B_{n r}(\tau) B_{n \pi}(\tau) g_{r} g_{\pi} \rho_{r \pi}+B_{n \pi}(\tau)^{2} g_{\pi}^{2}\right)
\end{aligned}
$$

In order to calculate the Inflation Index Bands (IIB) yield, we apply the Lema relation to the real price of the bonds and obtain the following model:

$$
\frac{d P_{r}\left(r_{t}, t, T\right)}{P_{r}\left(r_{t}, t, T\right)}=\mu_{n}(t, T-t) d t-B_{r r}(T-t) g_{t} d W_{t}^{r}
$$

Where:

$$
\left.\mu_{r}(t, \tau)=\frac{d}{d \tau} A_{r}(\tau)+\frac{d}{d \tau} B_{r r}(\tau) r_{t}-B_{r r}(\tau) k_{r} \overline{(r}-r_{r}\right)+\frac{1}{2} B_{r r}(\tau)^{2} g_{r}^{2}
$$

If we replace the Lema relation with the IIB price we will reach equality:

$$
P_{I}\left(r_{t}, I_{t}, t, T\right)=P_{r}\left(r_{t}, t, T\right) I_{t}
$$

As the price index ${ }^{I}{ }^{t}$ follows a chronological, dynamic course, we obtain the process of returning the IIB, according to the relation:

$$
\frac{d P_{I}\left(r_{t}, I_{t}, t, T\right)}{P_{I}\left(r_{t}, I_{t}, t, T\right)}=\mu_{I}(t, T-t) d t-B_{r r}(T-t) g_{r} d W_{t}^{r}+\sigma_{I} d W_{t}^{I}
$$

Where:

$$
\mu_{I}(t, \tau)=\mu_{r}(t, \tau)+\pi_{t}-B_{r r}(\tau) g_{r} g_{I} \rho_{I r},
$$

Checking for equality: $\sigma_{I r} d t=d W_{t}^{r} d W_{t}^{I}$

Finally, the yield of the real financial account ${ }^{M_{I}(t)}$ can be easily calculated using the adapted relation $M_{r}(t)=\exp \int_{0}^{t} r_{s}(d s)$, which becomes:

$$
\frac{d M_{I}(t)}{M_{I}(t)}=\left(r_{t}+\pi_{t}\right) d t+\sigma_{I} d W_{t}^{I}
$$

To obtain the price of the bond, we will use the standard without resorting to financial arbitrage. It turns out that the excess yield must be equal to the risk rates for the nominal bonds $W_{t}^{r}$ and $W_{t}^{\pi}$ and the real financial account, so that the relations are satisfied: 


$$
\begin{aligned}
& \mu_{n}(t, \tau)-R_{t}=-B_{n r}(\tau) g_{r} \Lambda_{t}^{r}-B_{r \pi}(\tau) g_{\pi} \Lambda_{t}^{\pi}, \forall \tau>0 \\
& \mu_{I}(t, \tau)-R_{t}=-B_{r r}(\tau) g_{r} \Lambda_{t}^{r}+\lambda_{1} \sigma_{1}, \forall \tau>0 \\
& \pi_{t}+r_{t}-R_{t}=\lambda_{1} \sigma_{1}
\end{aligned}
$$

Where $\mu_{n}(t, \tau), \mu_{I}(t, \tau)$ is defined in relations (1) and (7) being the market prices of the risk associated with the sources of risk $W_{t}^{r}, W_{t}^{\pi}$ and respectively $W_{t}^{I}$.

We suppose that the market prices of the risk for $W_{t}^{r}$ and $W_{t}^{\pi}$ are dependent on a factor, so we can formulate the relationships:

$$
\Lambda_{t}^{r}=\lambda_{0}+\lambda_{1} r_{t} \text { ssi } \Lambda_{t}^{\pi}=l_{0}+l_{1} r_{t}
$$

If equality without arbitration, according to relations (9) - (11) is satisfied, then:

- The $A_{n}(\tau), B_{n r}(\tau), B_{n \pi}(\tau)$ coefficients for nominal bond prices result from the relations:

$$
\begin{aligned}
& B_{n r}(\tau)=\frac{1}{\tilde{k}_{r}}\left(1-e^{-\tilde{k}_{r} \tau}\right), \quad \text { cu } \tilde{k}_{r}=k_{r}+g_{r} \lambda_{1} \\
& B_{n \pi}(\tau)=\frac{1}{\tilde{k}_{\pi \pi}}\left(1-e^{-\tilde{k}_{\pi \pi} \tau}\right), \quad \text { cu } \widetilde{k}_{\pi \pi}=k_{\pi \pi}+g_{\pi} \lambda_{1}
\end{aligned}
$$

and

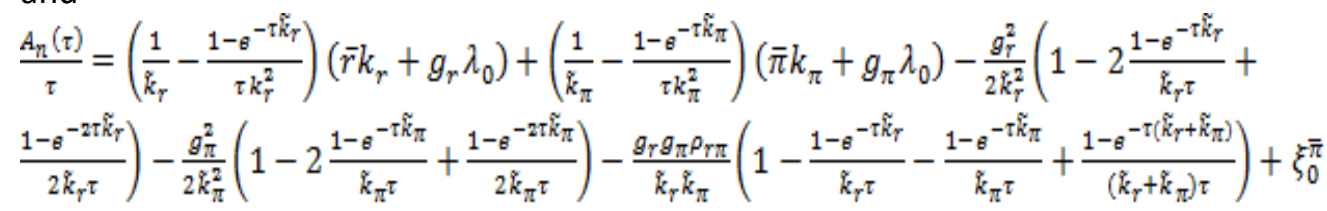

- The $A_{r}(\tau), B_{r r}(\tau)$ coefficients for real yield are determined using the following relations:

$B_{r r}(\tau)=\frac{1}{\tilde{k}_{r}}\left(1-e^{-\tilde{k}_{r} \tau}\right)$, cu $\tilde{k}_{r}=k_{r}+g_{r} \lambda_{1}$

and

$$
\frac{A_{r}(\tau)}{\tau}=\left(\frac{1}{\tilde{k}_{r}}-\frac{1-e^{-\tau \tilde{k}_{r}}}{\tau k_{r}^{2}}\right)\left(\bar{r} k_{r}-g_{r}\left(\lambda_{0}-\sigma_{I} \rho_{I r}\right)\right)-\frac{g_{r}^{2}}{2 \tilde{k}_{r}^{2}}\left(1-2 \frac{1-e^{-\tau \tilde{K}_{r}}}{\tilde{k}_{r} \tau}+\frac{1-e^{-2 \tau \tilde{k}_{r}}}{2 \tilde{k}_{r} \tau}\right)
$$

The market prices of the risk, according to the relation (12) are determined according to the case, being not constant. The ${ }^{B_{n r}}(\tau)$ coefficient represents the degree of sensitivity of the yield on bonds at the real interest rate $r_{t}$. Based on the yield formula according to the relation $Y_{n}(t, T)=\frac{-\ln P_{n}(t, T)}{T-t}=\frac{A_{n}(T-t)}{T-t}+\frac{B_{n r}(T-t)}{T-t} r_{t}+\frac{B_{n \pi}(T-t)}{T-t} \pi_{t}$, the sensitivity of the factor is calculated using the relation:

$$
\frac{\partial}{\partial r} Y_{n}\left(r_{t}, \pi_{t}, t, T\right)=\frac{B_{n r}(T-t)}{T-t}
$$

If the market price of the risk is constant, this means that $\lambda_{1}=0$ equivalent (13), then $\tilde{k}_{r}=k_{r}$. In this case, the parameter $k_{r}$ has a dual role: on the one hand $k_{r}$ determines the sensitivity of the bond yield factor, and on the other hand $k_{r}$ expresses the average inversion rate of the real rate dynamics determined by the relation $d r_{t}=k_{r}\left(\bar{r}-r_{r}\right) d t+g_{r} d W_{t}^{r}$.

If the pair parameters $\left(\xi_{0}^{\bar{\pi}}, \bar{\pi}\right){ }_{s, i}\left(\xi_{0}^{\hat{\pi}}, \hat{\pi}\right)$ satisfy the relation:

$$
\xi_{0}^{\bar{\pi}}+\bar{\pi}=\xi_{0}^{\hat{\pi}}+\hat{\pi}
$$


then each nominal yield model, according to the relationship $P_{n}\left(r_{t}, \pi_{t}, t, T\right)=\exp \left(-A_{n}(T-t)-B_{n r}(T-t) r_{t}-B_{n \pi}(T-t) \pi_{t}\right.$ with the coefficients obtained from (13), (14) and (15) for each pair of parameters, generates the same bond yield dynamics.

The nominal instantaneous interest rate $R_{t}$ is defined as an instantaneous yield of the form $R_{t}=\lim _{T \rightarrow t} Y_{n}(t, T)$ and it is calculated using the relation:

$$
R_{t}=\lim _{t \rightarrow T} Y_{t}(t, T)=r_{t}+\pi_{t}+\xi_{0}^{\bar{\pi}}
$$

It is inscribed in the condition without arbitration (11) and satisfies the equations $\xi_{0}^{\bar{\pi}}=A_{n}^{\prime}(0)=-\lambda_{I} \sigma_{I}$ by applying the L' Hospital rule.

If we change $\tau=0$ with any arbitrary number $\pi_{t}$ we just have to subtract the same sum from ${ }^{\bar{\pi}}$ so that equality (19) is maintained and $R_{t}$ remains thus unchanged. The real yield model excludes the possibility that two different sets of parameters have the same dynamic evolution. Therefore, the dynamics of the real yield is clearly identified by the real yields and the real rate ${ }^{r_{t}}$ is equal to the instantaneous real yield, respectively:

$$
r_{t}=\lim _{t \rightarrow T} Y_{t}(t, T)
$$

We specify, that by the demonstration performed in this model, we have based the model expressed at the price without arbitrage.

\section{market}

3.2. Some aspects regarding the effect of the dynamics of inflation in the study of the capital

The analysis of the capital market, especially the forecast of the market price cannot be done without taking into account the evolution of the dynamics of the price index. In other words, we will have to consider and estimate the dynamics of inflation. In the capital market, in an uncertain environment, which is influenced, most of the times, by the dynamics of inflation. As a result, the forecast of market prices will have to be studied in close agreement (correlation) with the price index. We know that a prospect of price changes causes turmoil in the asset market, from the point of view of sale or purchase. Of course, the market price fluctuates according to this trend.

In the following, we will try to build a statistical-econometric model that can be used in estimating the dynamics of inflation and its effect on capital market prices.

In this sense, we will consider in building a model, the dynamics of the price index based on the realized price index. Therefore, the dynamics can be calculated according to the relation:

$$
d \ln I_{t}=\left(\pi_{t}-\frac{\sigma_{I}^{2}}{2}\right) d t+\sigma_{I} d W_{t}^{I}
$$

If we use the Euler-Maruyama scheme, equality results:

$$
\ln I_{t+\Delta}-\ln I_{t}=\left(\pi_{t}-\frac{\sigma_{I}^{2}}{2}\right) \Delta+\sigma_{I}\left(W_{t+\Delta}^{I}+W_{t}^{I}\right)
$$

We consider that ${ }^{\pi_{t}}$, follows a normal dynamics.

The annualized inflation is based on the ratio $\left(\ln I_{t+\Delta}-\ln I_{t}\right) / \Delta$.

To estimate the process difficult to detect ${ }^{\pi_{t}}$ by discrete time observation of the price index $I_{t}$, we will resort to the "Kalman" filtering method, and the state equation will take the form: $r_{t+\Delta}=e^{-k_{r} \Delta} r_{t}+\bar{r}\left(1-e^{-k_{r} \Delta}\right)+\sigma_{r} u_{r t}$

It is interesting to compare the inflation rate achieved with the expected inflation, because the inflation rate is much more volatile than expected. We find that the estimated inflation rate fluctuates around the long-term average.

Therefore, the market price of the price index risk ${ }^{\lambda_{I}}$ is given by the following relation: 


$$
\lambda_{I}=-\frac{\xi_{0}^{\pi}}{\sigma_{I}}=-\frac{\xi_{0}^{0}-\bar{\pi}}{\sigma_{I}}
$$

If we calculate the correlation between $W_{t}^{I}, W_{t}^{r}$ and $W_{t}^{\pi}$, we find that $W_{t}^{r}$ and $W_{t}^{\pi}$ are monitored daily, while the estimated shock $W_{t}^{I}$ is monthly. But in order to calculate $\rho_{I r}, \rho_{I \pi}$ we have to record for $W_{t}^{r}$ and $W_{t}^{\pi}$ the monthly evolution.

The negative evolution $\rho_{\text {Ir }}$ compared to the inflation shock achieved is negatively correlated with the real interest rate, which increases due to the estimated inflation rate. After estimating the correlation, using concrete results, we can calculate the market price of the real interest rate risk through the relation:

$$
\lambda_{0}=\lambda_{0}^{*}+\sigma_{I} \rho_{I r}
$$

In order to stimulate the dynamics of the yield we must take into account the situation of asset allocation, there being an asset of shares in the set of investment opportunities. Applying the formula to the dynamics of the stock price, we obtain the equivalent expression, from the relation:

$$
d \ln P_{S}(t)=\left(R_{t}+\lambda_{S} \sigma_{S}-\frac{\sigma_{S}^{z}}{2}\right) d t+\sigma_{S} d W_{t}^{S}
$$

The estimation model is obtained, using "Eular-Maruyama" approximation methods, which we apply to continuous time dynamics with a range of $\Delta_{t}=\frac{1}{250}$.

For the issue of asset allocation, we must also know the correlations between $W_{t}^{S}, W_{t}^{r}, W_{t}^{\pi} s W_{t}^{I}$ shocks. In the case of optimal intertemporal portfolios, we will study a set of investment opportunities that will include four components for risky assets, namely a short-term nominal bond, a six-month rate, a longterm nominal bond and a long-term indexed bond.

If we study the dependence of the optimal investment strategy on the risk aversion that the investments made by agents have, we obtain the parameter ${ }^{r_{i}}$. Therefore, the higher the ${ }^{r_{i}}$ the greater the risk aversion. If the time horizon is high, say ten years and there will be no restrictions on short-term market sale, things seem normal. As risk aversion grows, investors shift their level of capital to inflationindexed bonds.

It is worth noting that inflation-indexed bonds are chosen to mature at the end of the investment horizon. Therefore, the asset offers a fixed payment as the investment horizon matures. The results indicate that as the ${ }^{r_{i}}$ increases, the proportion of IIB investments approaches $100 \%$ and, at the same time, the other investments decrease to zero. If we analyze how the strategy of asset allocation varies according to the investment horizon, in the real market, the investment horizon varies over a long period, from two to twelve years. The short-term nominal bond matures six months, while the long-term nominal bond and inflation-indexed bonds mature at the end of the investment. In order to prevent the holding of extreme investments, we will choose a number higher than ${ }^{r_{i}}$. The agents prefer to hold the nominal bonds (NB-T) at maturity, at the end of the investment, in order to have a fixed payment. At the point where the investment horizon is maximum ( $T=10$ years), the investors' reaction becomes prudent.

\section{Conclusions}

In this article, made after the study undertaken by the authors, regarding the capital market, from a careful interpretation we can draw a series of theoretical and practical conclusions. First of all, we find that the capital market must be analyzed taking into account the inflation rate factor, which has a special effect on the analysis of portfolios, the accumulation of assets, the portfolio decisions, taken in the market, taking into account the risk of inflation. From the presented study it is very clear that by statistical-econometric methods, expressed by matrix relations, parameters can be calculated and the evolution of the capital market under inflation risks can be identified. Statistical-econometric relations are used to highlight the prospects of evolution, based on parameters that ensure the estimation of future developments in the short, medium and long term, the dynamics of inflation, as well as the evolution of yield. 
The risk of inflation is an important factor, which must be taken into account in any analysis that is carried out in relation to the capital market and its dynamic evolution.

On the other hand, it comes to the conclusion that estimating the dynamics of inflation in the analysis of the capital market is a determining factor, which should not be neglected in any study on the forecast, the forecast of the capital market. Inflation does not happen by chance, but is determined by certain factors, which impose a certain risk of its evolution. Therefore, placing assets on the capital market, investments, must take into account the way in which both economic factors and inflation dynamics evolve. These two factors clearly have an inverse correlation, in the sense that, the higher the dynamics of inflation, the more prospects for the evolution of the capital market, as yield, are smaller, lower. Inflation is the result of rising prices that act differently depending on the market situation.

From a practical point of view, it is considered that a used model has the effect in identifying through the calculated parameters, a real estimation of the perspective of the evolution of the capital market and on the consequence of the return of the capital market.

\section{References}

1. Anghelache, C., Anghel, M. G. (2018), Econometrie generala. Teorie și studii de caz. Editura Economica, Bucureşti.

2. Arcidiacono, P., Miller, R. A. (2011), Conditional Choice Probability Estimation of Dynamic Discrete Choice Models with Unobserved Heterogeneity. Econometrica, 79 (November 2011), 1823-1867.

3. Brennan, M. J., Xia, Y. (2002), Dynamic asset allocation under inflation, Journal of Finance, 57 (3), 1201-1238.

4. Cooper, I. (2009), Time-Varying Risk Premiums and the Output Gap. Review of Financial Studies, Society for Financial Studies, 22 (7): 2601-2633.

5. Dobrodolac, T. (2011), Forecasting by econometric models as support to management, Perspectives of Innovations, Economics \& Business, 7 (1), 72-76.

6. Dougherty, C. (2008), Introduction to econometrics. Fourth edition, Oxford University Press.

7. Elliott, G., Müller, U. K., Watson, M. W. (2015), Nearly Optimal Tests When a Nuisance Parameter is Present Under the Null Hypothesis. Econometrica, 83, 771-811.

8. Ferreira, M. A., Santa-Clara, P. (2011), Forecasting stock market returns: The sum of the parts is more than the whole. Journal of Financial Economics, 100; 514-537.

9. Giacomini, R., Rossi, B. (2010), Forecast comparisons in unstable environments. Journal of Applied Econometrics, John Wiley \& Sons, Ltd., 25(4), 595-620.

10.Hjalmarsson, E. (2010), Predicting Global Stock Returns. Journal of Financial and Quantitative Analysis, Cambridge University Press, 45(1): 49-80.

11.Jarrow, R., Yildirim, Y. (2003), Pricing treasury inflation protected securities and related derivations using an HJM model. Journal of Financial and Quantitative Analysis, 38(2), 337-358.

12. Markowitz, H. (2014), Mean-variance approximations to expected utility. European Journal of Operational Research, 234, 346-355.

13.Müller, U. K. (2007), A Theory of Robust Long-Run Variance Estimation. Journal of Econometrics, 141, 1331-1352.

14.Piketty, T. (2014), Capital in the twenty-first century, Boston: Harvard University Press.

15.Rapach, D. E., Zhou, G. (2013), Forecasting stock returns, In Handbooks of economic forecasting (Vol. 2, in press), Elsevier.

16.Wachter, J. (2003), Risk avesion and allocation to long-term bonds. Journal of Economic Theory, $112,325-333$.

17.Welch, I., Goyal, A. (2008), A comprehensive look at the empirical performance of equity premium prediction. Review of Financial Studies, 21 (4), 1455-1508.

18.Zhou, G. (2010), How much stock return predictability can we expect from an asset pricing model?. Economics Letter, 108, 184-186. 